\title{
Derivation of the Quantum Probability Rule without the Frequency Operator
}

\author{
Sumio Wada \\ Institute of Physics \\ The University of Tokyo, Komaba \\ Tokyo, 153-8902, Japan
}

October 31, 2018

\begin{abstract}
We present an alternative frequencists' proof of the quantum probability rule which does not make use of the frequency operator, with expectation that this can circumvent the recent criticism against the previous proofs which use it. We also argue that avoiding the frequency operator is not only for technical merits for doing so but is closely related to what quantum mechanics is all about from the viewpoint of many-world interpretation.
\end{abstract}

KEYWORDS:quantum probability rule, Born's rule, relative frequency, frequency operator, outcomes of measurements, wave function, quantum postulate, many-world interpretation, realism, determinism

\section{Introduction}

Whether the quantum probability rule (QPR), or Born's rule, should be postulated as one of axioms of quantum mechanics has been a long standing issue since the authors of refs.1) and 2) claimed that this rule can be derived by reinterpretting the probability as the frequency of outcomes of measurements. They introduced a so-called frequency operator, which expresses the relative frequency (the proportion of the frequency) of a certain specific outcome in the infinite repetition of measurements, and claimed that a infinite repetition state is an eigenstate of this operator with the eigenvalue which QPR dictates. However it was argued that their statement is about many but not infinite repetition of measurements and did not constitute a rigorous proof that the infinite repetition state is an eigenstate. Their proof was claimed to correspond to the weak law of large number in the classical probability theory.

*Electric address: wada@hep1.c.u-tokyo.ac.jp 
In refs.3) and 4), the authors improved this situation. They defined the frequency operator acting on the infinite repetition state, and proved what amounts to the strong law of large number. But in the course of the proof they had to introduce the measure, i.e. the weight of each infinite sequence of outcomes. Recently, in ref.5), it was pointed out that the measure and so the frequency operator itself is not unique and, as a result, we can arbitrarily changes its eigenvalue. Adopting a specific form of the measure amounts to adopting an assumption about the probability rule from the outset. If so, the claim that QPR can be derived from the frequency becomes dubious.

What we try to do in this paper is to prove QPR, sticking to frequencists' approach but without recourse to the frequency operator. We circumvent the above criticism by avoiding the frequency operator, but we also take note that there is a more fundamental reason to avoid the frequency operator.

In the orthodox formulation of quantum mechanics, beside the probability rule, the following ansatz is often postulated.

Postulate: Every observable is associated with a Hermitian operator $O$. Only possible outcomes of a measurement of $O$ are eigenvalues of $O$. When a system is in an eigenstate $\mid \psi>$ of $O$, i.e. $O|\psi>=\lambda| \psi>$, then a measurement of $O$ will yield the value $\lambda$.

This is the reason why the frequency operator $\mathrm{F}$ was introduced. If $\mathrm{F}$ were unambiguously defined and a certain state were an eigenstate of the $\mathrm{F}$ with the eigenvalue $\mathrm{p}$, then it would follows from the above that the relative frequency in which a certain outcome occurs would be p.

I challenge this type of reasoning from the viewpoint of a many-worlder and quantum cosmologist. Any measurement is a phenomenon which occurs in the Universe, and as such, should be expressed in the state of the world (i.e. in the wave function of the Universe). Mathematically we may be able to devise an operator by which we can investigate a property of the state, but that should not be required.

As a simple example, suppose that a wave function $\psi(q)$ has a form

$$
\psi(q) \propto \delta\left(q-q_{0}\right)
$$

where $\mathrm{q}$ is a c-number variable and $q_{0}$ is a certain constant. This wave function vanishes unless $q=q_{0}$ and so it should be natural to claim that $q=q_{0}$ in this state.

However, if we stick to the above postulate, we have to introduce the operator $\hat{q}$ which satisfies

$$
\hat{q} \psi(q)=q_{0} \psi(q)
$$

and deduce that the outcome of measurements of the observable $\hat{q}$ should be $q_{0}$. One may wonder whether this procedure is really necessary. If we are concerned with the outcome of the measurement, then we introduce the wave function $\Psi$ of the apparatus whose readout $Q$ is in one-to-one correspondence with $q$. Then, if the measurement is accurate, we will get

$$
\Psi(Q) \propto \delta\left(Q-Q_{0}\right)
$$


from which we should be able to deduce that $Q=Q_{0}$ (and so $q=q_{0}$ ) without recourse to any operator.

In the above simple example the distinction between eq.(1) (or eq.(3)) and eq.(2) looks to be practically irrelevant. However, when we seriously ponder over what wave functions actually mean and, in particular, when the definition of the operator itself is ambiguous, the distinction may not be an irrelevant issue anymore.

In this paper we insist that the place to look for predictions in quantum theory is not an operator (nor an observable) but a wave function. An operator is regarded as no more than a means to extract information when convenient. It is from this viewpoint that we reexamine frequencists' derivation of QPR and try to derive the same conclusion without recourse to the frequency operator.

\section{Naive Derivation}

The difficulty in this problem lies in deriving information about the infinite repetition of measurements from considerations on many but finite repetition of measurements. In this section we present a "proof" of QPR without a frequency operator but will take a rather naive attitude on this point, optimistically assuming that a limit of finite repetitions might give the required result about the infinite repetition. (The calculation shown below was presented before in ref.6).

We start from a finite repetition state

$$
\left|\Psi>_{N}=\right| \psi, 1>|\psi, 2>| \psi, 3>\ldots \mid \psi, N>
$$

Each element $\mid \psi, i>$ is supposed to represent a single identical particle with spin, each of which is prepared in a spatially different position but is supposed to be in an identical state as long as the spin is concerned. When this particle is a fermion, the whole state should be antisymmetrized, but this requirement is assumed to be taken care of by the spatial part of the wave function. The spin part of the wave function, which is of our concern in the following argument, is symmetric because all the spin states are identical. For simplicity, $\mid \Psi>_{N}$ is supposed to represent only the spin part of the state.

Because all the spins are assumed to be in an identical state, we can write as

$$
|\psi, i>=a| \alpha, i>+b \mid \beta, i>
$$

where and represent a spin state while $a$ and $b$ are $i$-independent coefficients with

$$
|a|^{2}+|b|^{2}=1
$$

We substitute eq.(5) into eq.(4) and expand it. We get various terms, each of which has certain numbers of $\mid \alpha>$ and $\mid \beta>$. When the number of $|\alpha\rangle$ is $n$, the number of $\mid \beta>$ is $N-n$ and the relative frequency $\mathrm{r}$ (of $\mid \alpha>$ ) is defined as

$$
r=n / N
$$


Next we introduce the normalized and symmetrized state $|r\rangle$ with a fixed relative frequency, which is

$$
\mid r>_{N} \equiv_{N} C_{n}^{-1 / 2}\{|\alpha, 1>\ldots| \alpha, n>|\beta, n+1>\ldots| \beta, N>+ \text { perm. }\}
$$

The set $\{\mid r>: r=n / N, n=1 \sim N\}$ is complete in the space of symmmetrized $N$-copy states, and we can expand $\mid \Psi>_{N}$ in terms of $\mid r>$ as

$$
\left|\Psi>_{N}=\sum_{r} c_{N}(r)\right| r>_{N}
$$

where

$$
c_{N}(r)={ }_{N} C_{n}^{1 / 2} a^{n} b^{N-n}
$$

It is easy to show that

$$
\begin{gathered}
\sum\left|c_{N}(r)\right|^{2}=1 \\
\sum\left(r-|a|^{2}\right)^{2}\left|c_{N}(r)\right|^{2}=|a|^{2}|b|^{2} / N(\rightarrow 0 \text { as } N \rightarrow \infty)
\end{gathered}
$$

These results imply that $\left|c_{N}(r)\right|^{2}$ concentrates at $r=|a|^{2}$ in the limit $N \rightarrow \infty$.

Note in passing that the above calculation with the two-level systems can be easily generalized to multi-level systems and also to continuous variable cases, as are shown in Appendices.

\section{An Alternative Postulate of Quantum Mechan- ics}

The above results tempt us to conclude that the relative frequency of the outcome in the infinite repetition of spin measurements is $|a|^{2}$, as QPR dictates. If this claim can be justified, we need not to include QPR in the set of axioms (postulates) of quantum mechanics, although we do need some alternative postulate, which will be weaker than QPR, to justify the above argument. In this section we investigate what we actually need, propose a weaker postulate and modify the above argument accordingly.

We start from a trivial case in which the wave function $\psi$ is a function of a discrete variable $n$ and is proportional to Kronecker's delta,

$$
\psi(n) \propto \delta_{n n_{0}}
$$

Naturally we conclude that the value of $n$ in this state is $n_{0}$. Its extension to a continuous variable (say $q$ ) might be

$$
\psi(q) \propto \delta\left(q-q_{0}\right)
$$

in which case we would claim that $q=q_{0}$. However this reasoning can not be applied to $c(r)$ (which is the $N \rightarrow \infty$ limit of $c_{N}(r)$ in the previous section). While $\delta\left(q-q_{0}\right)$ is not normalizable (not square-integrable), $c(r)$ was normalized. What behaves like a delta function is not $c(r)$ but $|c(r)|^{2}$. 
Normalizable functions are more legitimate states in an ordinary rigorous treatment of quantum mechanics, and so, any postulate of the quantum theory should be at least applicable to them. It should also be applicable whether a variable is discreet or continuous. We will pursue such an extension of eq.(13).

First we note that, judging from the situation for $c(r)$, natural extension of eq.(13) to continuous variable cases should not be eq.(14), but something which involves $|\psi(q)|^{2}$. (Note that $\psi(n) \propto \delta_{n n_{0}}$ and $|\psi(n)| 2 \propto \delta_{n n_{0}}$ are equivalent in discreet variable cases.)

Let us start from a simple example. We consider a space of functions $\psi(q)$ in which the inner product is written as

$$
\sum \psi_{1}(q)^{*} \cdot \psi_{2}(q)
$$

A variable $q$ is assumed to be real and the summation is over all possible values of $q$. $q$ can be either discrete or continuous. When $q$ is continuous, the summation in the above equation is replaced with an integral.

For later convenience we introduce the following notation

$$
\begin{gathered}
\rho_{q}\left(\psi_{1}, \psi_{2}\right) \equiv \psi_{1}(q)^{*} \cdot \psi_{2}(q) \\
\rho_{q}(\psi) \equiv|\psi(q)|^{2}
\end{gathered}
$$

$\rho_{q}(\psi)$ is nothing but $\rho_{q}\left(\psi_{1}, \psi_{2}\right)$ with $\psi_{1}=\psi_{2}=\psi$.

$\psi(q)$ can be interpreted as a $q$-representation of a state $|\psi\rangle$, that is

$$
\psi(q)=<q \mid \psi>
$$

and

$$
\rho_{q}\left(\psi_{1}, \psi_{2}\right)=<\psi_{1}|q><q| \psi_{2}>
$$

Then the inner product eq.(15) means

$$
<\psi_{1}\left|\psi_{2}>\equiv \sum<\psi_{1}\right| q><q \mid \psi_{2}>
$$

which implies the completeness relation

$$
1=\sum|q><q|
$$

We assume that a quantum state $|\psi\rangle$ is represented by a normalized vector,. which should be one of the axioms of quantum theory. This means

$$
\sum \rho_{q}(\psi)=<\psi \mid \psi>=1
$$

Suppose that for any positive number

$$
\begin{gathered}
\sum_{q<q_{0}-\epsilon} \rho_{q}(\psi)=0 \\
\sum_{q<q_{0}+\epsilon} \rho_{q}(\psi)=1
\end{gathered}
$$


Then we naturally conclude that the value of $q$ in this state is $q_{0}$. These conditions are essentially equivalent to say that $\rho_{q}=\delta_{q q_{0}}$ or $\rho_{q}=\delta\left(q-q_{0}\right)$, but we prefer the above expression to avoid arguments about what the delta function actually means.

In general the above conditions do not hold and the state $\psi$ does not take a specific value of $q$. However we may try another variable, say $\tilde{q}$, in which case we should examine $\rho_{\tilde{q}}(\psi)$, i.e. the $\tilde{q}$ version of $\rho_{q}(\psi)$. If there is a completeness relation analogous to eq.(21) for $\tilde{q}$

$$
1=\sum|\tilde{q}><\tilde{q}|
$$

then we can follow the same argument above. We define $\rho_{\tilde{q}}(\psi)$ and examine whether the conditions similar to eqs. $(23,24)$ hold or not for a certain value of $\tilde{q}_{0}$.

However we can not use this approach either to derive QPR from the result in the previous section. Suppose that we are working in the (non-separable) space spanned by the infinite repetition states. The state defined in eq.(8), which has a fixed relative frequency $r$, is a state with a finite number of copies and so does not belong to this space, which means that we can not utilize the completeness relation to define what is supposed to become $\rho_{r}(\Psi)$.

However we can define the quantity which is very close to that. Write a state with infinite number of copies of a two-level system as

$$
\left|\Psi>_{\infty}=\right| \psi, 1>|\psi, 2>\ldots| \psi, N>\times \mid \psi, N+1>\ldots\left(\equiv\left|\Psi>_{N} \times\right| \Psi>_{\infty-N}\right)
$$

$\psi$ in each element does not need to coincide with each other at this stage but eventually we restrict $|\Psi\rangle_{\infty}$ to the inifinite repetition state.

We define the inner product of two of such states as

$$
\infty<\Psi_{1}\left|\Psi_{2}>_{\infty} \equiv \prod_{i}<\psi_{1}, i\right| \psi_{2}, i>
$$

$\mid \Psi>_{\infty}$ is normalized when all of $|\psi, i\rangle^{\prime} s$ are normalized. When $\mid \Psi_{2}>_{\infty}$ is a linear combination of two such states as

$$
\left|\Psi_{2}>_{\infty}=a\right| \Psi_{2 a}>_{\infty}+b \mid \Psi_{2 b}>_{\infty}
$$

then

$$
\infty<\Psi_{1}\left|\Psi_{2}>_{\infty} \equiv a \prod_{i}<\psi_{1}, i\right| \psi_{2 a}, i>+b \prod_{i}<\psi_{1}, i \mid \psi_{2 b}, i>
$$

Next we define a $r$-dependent bilinear functional of $\mid \Psi>_{\infty}$ as

$$
\rho_{r, N}\left(\Psi_{1}, \Psi_{2}\right)=_{N}<\Psi_{1}\left|r>_{N} \cdot{ }_{N}<r\right| \Psi_{2}>_{N} \times_{\infty-N}<\Psi_{1} \mid \Psi_{2}>_{\infty-N}
$$

where $\mid r>_{N}$ is what was defined in eq.(8) in the previous section.

Note that, in the above equation, $r$ should be a rational number $(0<r<1)$ for which $r N(=n)$ is an integer. For a general real number $r(0<r<1)$ we 
consider a sequence $\left\{r_{N}: N=1 \sim \infty, r_{N} N=\right.$ integer $\}$ in which $r_{N}$ is the possible rational number which is nearest to $r$ and we define

$$
\rho_{r}\left(\Psi_{1}, \Psi_{2}\right) \equiv \lim _{N \rightarrow \infty} N \rho_{r_{N}, N}\left(\Psi_{1}, \Psi_{2}\right)
$$

We multiplied by $N$ on the right-hand side to take into account the fact that $r$ is a continuous variable while $r_{N}(=n / N)$ is discrete and $\Delta n=N \Delta r$. This definition makes it possible to require

$$
\int_{0<r<1} \rho_{r}\left(\Psi_{1}, \Psi_{2}\right) d r=\lim _{N \rightarrow \infty} \sum_{r_{N}} \rho_{r_{N}, N}\left(\Psi_{1}, \Psi_{2}\right)
$$

For general states $\Psi$, the limit in eq.(31) does not necessarily exist, but does exist for the states which we are actually interested in, namely the infinite repetition states. In the following we restrict $\Psi$ to these states (and their linear combinations).

Now we examine the case that $\Psi_{1}=\Psi_{2}(\equiv \Psi)$ are infinite repetition states in which all of their elements $\mid \psi, i>$ are expressed as in eq.(5). Then, from the result in the previous section, it follows that

$$
\rho_{r_{N}, N}(\Psi)=\left|c_{N}\left(r_{N}\right)\right|^{2}
$$

(As before, when $\Psi_{1}=\Psi_{2}=\Psi$, we write $\rho\left(\Psi_{1}, \Psi_{2}\right)$ simply as $\rho(\Psi)$.) We can show that its limit

$$
\rho_{r}(\Psi) \equiv \lim _{N \rightarrow \infty} N \rho_{r_{N}, N}(\Psi)
$$

satisfies the conditions of the form eqs. $(23,24)$, which means that, for any arbitrary positive number of,

$$
\begin{aligned}
& \int_{0<r_{0}-\epsilon} \rho_{r}(\Psi) d r=0 \\
& \int_{r<r_{0}+\epsilon} \rho_{r}(\Psi) d r=1
\end{aligned}
$$

(where $r_{0} \equiv|a|^{2}$ ). To prove them, first note that these equations are equivalent to

$$
\begin{aligned}
& \lim \sum_{r<r_{0}-\epsilon} \rho_{r, N}(\Psi)=0 \\
& \lim \sum_{r>r_{0}+\epsilon} \rho_{r, N}(\Psi)=0
\end{aligned}
$$

The first identity, for an example, holds because

$$
\sum_{r<r_{0}-\epsilon} \rho_{r, N}(\Psi)<1 / \epsilon^{2} \sum_{r<r_{0}-\epsilon}\left(r_{0}-r\right)^{2} \rho_{r, N}(\Psi)=1 / \epsilon^{2} \cdot|a|^{2}|b|^{2} / N
$$

The right-hand side vanishes in the limit $N \rightarrow \infty$ with fixed.

Now we have understood what we need to derive QPR. We summarize it as a new postulate of quantum theory as follows: 
Postulate: Suppose that $\rho_{q}\left(\psi_{1}, \psi_{2}\right)$ be a certain bilinear functional of the quantum state $\psi_{1}$ and $\psi_{2}$ (linear in $\psi_{2}$ and antilinear in $\psi_{1}$ ) which depends on a real variable q (either discrete or continuous) and reproduces the inner product of the two states when they are summed over all possible values of $\mathrm{q}$

$$
\sum \rho_{q}\left(\psi_{1}, \psi_{2}\right)=<\psi_{1} \mid \psi_{2}>
$$

Let $\rho_{q}(\psi)$ be the case in which $\psi_{1}=\psi_{2}=\psi$ with $\psi$ normalized. If for a certain fixed value $q_{0}$ and any positive number

$$
\begin{gathered}
\sum_{q<q_{0}-\epsilon} \rho_{q}(\psi)=0 \\
\sum_{q<q_{0}+\epsilon} \rho_{q}(\psi)=1
\end{gathered}
$$

then we can conclude that the value of $q$ in the state $\psi$ is $q_{0}$. iWhen the variable $q$ is continuous, all the summations above are replaced with the integrals. Note also that it is not necessary to require that can be defined for arbitrary quantum state .)

Note that our $\rho_{r}(\Psi)$ defined in eq.(34) satisfies all the conditions of the above postulate in the space spanned in the infinite repetition states. Therefore we conclude that QPR can be derived from this postulate.

Note also that this postulate supersedes another postulate mentioned in section 1 about observables. Information about quantum states should be obtained not from operators but from wave function-based quantities (such as $\rho$ ). It does not matter which method we adopt if both procedures can be shown to be equivalent, which is the case in ordinary applications of quantum mechanics. But it does not seem to be the case for the relative frequency of infinite repetition states.

\section{Measurements of Many but Finite times}

The discussion above tells us what the relative frequency of a certain individual state should be in an infinite repetition state. If accurate measurement could be carried out for all the infinite individual states, then it would also tell us what the relative frequency of a certain outcome should be. In practice, however, we can not carry out measurements infinite times. For the outcome of measurement of, say, a hundred times the above argument itself does not tell us anything specific. If the result is very different from the prediction for the infinite measurement, we would feel uncomfortable and would think that something might be wrong. But can we say something more quantitative about that?

Let us consider an infinite repetition of a hundred measurement. We already know that a hundred repetition state can be expanded as in eq.(9), which we 
rewrite here as

$$
\left|\Psi>_{100}=\sum_{n} c_{100}(n)\right| n>_{100}
$$

where $N=100$ and $n=r N$ is an integer from 0 to 100 . If we apply QPR here, then we would tells that the probability that $|\alpha\rangle$ is observed $\mathrm{n}$ times (out of a hundred measurements) is $\left|c_{100}(n)\right|^{2}$. In our approach in which QPR is replaced with the postulate of the previous section, we have to consicer an infinite repetition of a hundred measurements. We can tell that, when a hundred measurements is repeated infinite times, then the relative frequency of the case that $\mid \alpha>$ is observed $\mathrm{n}$ times (out of 100 measurements) is $\left|c_{100}(n)\right|^{2}$. (To prove this statement we should generalize the two-level system in section 2 to the 101-level system, which is done in Appendix 1.) How small (large) it is determines how uncomfortable (or comfortable) we should feel when we get a certain outcome for a hundred measurement.

\section{Philosophical Aspects}

Classical mechanics is regarded as a theory of realism and determinism, while quantum mechanics has been often formulated as a theory of positivism and probability. Many people (including Einstein) has felt it unsatisfactory that fundamental laws of the Nature do not appear to describe the reality itself, but rather concerns about human perception of the Nature.

There are two aspects in quantum theory which cause us to suspect that quantum mechanics may not a theory about reality. They are QPR and the reduction of the state. If the frequencists' approach can be justified as has been advocated here, every reference to the probability in quantum theory is replaced with the word "frequency". We can totally get rid of the probability from the theory. It has also been pointed out in the context of the many world interpretation that we can get rid of the reduction of the state with the help of decoherence.

If this program turns out to be successful, its philosophical implications, especially its ontological meanings should be profound. We will come to be able to claim that quantum mechanics is a theory about the (not hidden but manifestly overt) reality. However we should take note that this reality can not be that of classical physics but that of quantum mechanics. This should be the reality which can be superposed and coexist in multiples. The interpretation of the reality as such might even justify the postulate proposed in this paper, but we would like to refrain from speculating about philosophical issues further here.

\section{Acknowledgement}

The author is indebted to Colin Bruce who brought his attention to ref.5).

Appendix 1: General Case 
In section 2 we presented an example of the decomposition of the infinite repetition states in terms or fixed frequency states by using the two-level system. Here we will show that the calculation can be generalized to general multi-level cases.

We are dealing with a symmetric component of the repetition state and so it is convenient to work with the bosonic creation operators such as

$$
\alpha^{+}|0>=| \alpha>\text {,etc. }
$$

Then in our two-level system in section 2 the N-copy state with a fixed relative frequency $r$ of is written as

$$
\left|r>=(1 / \sqrt{n !} \sqrt{(N-n) !}) \alpha^{+n} \beta^{+N-n}\right| 0>
$$

and so the repetition state $\mid \Psi>_{N}$ is expanded as

$$
\begin{gathered}
\left|\Psi>_{N}=1 / \sqrt{N !}\left(a \alpha^{+}+b \beta^{+}\right)^{N}\right| 0>=\sum 1 / \sqrt{N !}{ }_{N} C_{n} a^{n} b^{N-n} \alpha^{+n} \beta^{+N-n} \mid 0> \\
=\sum \sqrt{N ! / n !(N-n) !} a^{n} b^{N-n} \mid r>
\end{gathered}
$$

which reproduces eqs. $(9,10)$.

We generalize to the case in which the single particle state with $M$ levels is

$$
\left|\psi>=\sum_{i=1}^{M} a_{i} \alpha_{i}^{+}\right| 0>
$$

This is normalized as

$$
\sum\left|a_{i}\right|^{2}=1
$$

Next we consider the $N$-copy state in which each state $\alpha_{i}^{+} \mid 0>$ have a fixed relative frequency $r_{i}\left(\sum r_{i}=1\right.$ or alternatively $\sum n_{i}=N$ where $\left.r_{i}=n_{i} / N\right)$

$$
\left|\left\{r_{i}\right\}>=\prod_{i} 1 / \sqrt{n_{i} !} \alpha_{i}^{+n_{i}}\right| 0>
$$

Then the $N$-copy repetition state is decomposed as

$$
\begin{gathered}
\left|\Psi>_{N} \equiv 1 / \sqrt{N !}\left(\sum_{i} a_{i} \alpha_{i}^{+}\right)^{N}\right| 0>=\sum_{\left\{n_{i}\right\}} 1 / \sqrt{N !}{ }_{N} C_{\left\{n_{i}\right\}} \prod_{i} a_{i}^{n_{i}} \alpha_{i}^{+n_{i}} \mid 0> \\
=\sum \sqrt{N ! / \prod n_{i} !} \prod a_{i}^{n_{i}} \mid\left\{r_{i}\right\}>
\end{gathered}
$$

where we used the notation

$$
{ }_{N} C_{\left\{n_{i}\right\}}=N ! / \prod_{i} n_{i} !
$$

What corresponds to $c_{N}(r)$ in the two-level case is

$$
c_{N}\left(\left\{n_{i}\right\}\right)=\sqrt{N ! / \prod n_{i} !} \prod a_{i}^{n_{i}}
$$


Then it is straightforward to prove that

$$
\begin{gathered}
\sum_{\left\{n_{i}\right\}}\left|c_{N}\left(\left\{n_{i}\right\}\right)\right|^{2}=1 \\
\sum_{\left\{n_{i}\right\}}\left(r_{i_{0}}-\left|a_{i_{0}}\right|^{2}\right)^{2}\left|c_{N}\left(\left\{n_{i}\right\}\right)\right| 2=\left|a_{i_{0}}\right|^{2}\left(1-\left|a_{i_{0}}\right|^{2}\right) / N
\end{gathered}
$$

where $i_{0}$ is any fixed integer between 1 and $M$ and $r_{i_{0}}=n_{i_{0}} / N$. This means that

$$
\rho_{r_{i_{0}}, N}(\Psi)=\sum_{n_{i_{0}} \text { fixed }} N\left|c_{N}\left(\left\{n_{i}\right\}\right)\right| 2
$$

behaves like $\delta\left(r_{i_{0}}-\left|a_{i_{0}}\right|^{2}\right)$ in the limit $N \rightarrow \infty$. Then $\rho_{r_{i_{0}}}$ is defined as the limit $N \rightarrow \infty$ of $\rho_{r_{i_{0}}, N}$ and can be used to obtain the required prediction $r_{i_{0}}=\left|a_{i_{0}}\right|^{2}$, as was done in section 3 .

Appendix 2: Continuous Variables

We briefly mention the continuous variable case, taking quantum mechanics in one-dimensional space as an example. First we define a single particle state

$$
\left|\psi>=\int \psi(x) A^{+}(x)\right| 0>
$$

where $A^{+}(x)$ is an operator which creates a particle at x. The c-number wave function $\psi(x)$ is normalized as

$$
\int|\psi(x)|^{2}=1
$$

Let $\Delta$ be a region in the $\mathrm{x}$ space and define

$$
\begin{gathered}
\int_{\Delta}|\psi(x)|^{2} \equiv|a|^{2} \\
\int_{\Delta_{c}}|\psi(x)|^{2} \equiv|b|^{2}\left(=1-|a|^{2}\right)
\end{gathered}
$$

The N-copy state

$$
\left|\Psi>_{N}=1 / \sqrt{N !}\left\{\int \psi(x) A^{+}(x)\right\}^{N}\right| 0>
$$

is a superpostion of states each of which has a certain number, say n, of particles inside $\Delta$ and other particles outside it (inside $\left.\Delta_{c}\right) . H(N, n)$ be a space of such states, and $P_{N, n}$ be a projection operator which projects $\mid \Psi>_{N}$ on $H(N, n)$. Then

$$
{ }_{N}<\Psi\left|\Psi>_{N}=\sum_{n}{ }_{N}<\Psi\right| P_{N, n} \mid \Psi>_{N}=1
$$


Some calculation tells us that

$$
{ }_{N}<\Psi\left|P_{N, n}\right| \Psi>{ }_{N}={ }_{N} C_{n}|a|^{2 n}|b|^{2(N-n)}
$$

This expression is nothing but the square of eq.(10) and so we can conlude that, in the limit $N \rightarrow \infty, r=n / N=|a|^{2}$, as is expected. Note that this@method can also be applied to discreet multi-level cases.

\section{References}

1) D.Finkelstein: Transactions of the New York Academy of Sciences 25, 621 (1963).

2) J.B.Hartle: Am. J. Phys. 36, 704 (1968).

3) E.Fahri, J.Goldstone, and S.Gutmann: Ann. Phys. 192, 368 (1989).

4) J.Gutmann: Phys. Rev. A 52, 3560 (1995).

5) C.M.Caves and R.Schack: quant-ph/0409144v3 (2005).

6) S.Wada: Butsuri 44, (1989) 393 [in Japanese]. 